\title{
Changes in drug sensitivity and anti-malarial drug resistance mutations over time among Plasmodium falciparum parasites in Senegal
}

Daria Van Tyne ${ }^{1 \dagger}$, Baba Dieye ${ }^{2 \dagger}$, Clarissa Valim ${ }^{1,3+}$, Rachel F Daniels ${ }^{1 \dagger}$, Papa Diogoye Sène ${ }^{2}$, Amanda K Lukens ${ }^{1,3}$, Mouhamadou Ndiaye ${ }^{2}$, Amy K Bei ${ }^{1}$, Yaye Die Ndiaye ${ }^{2}$, Elizabeth J Hamilton ${ }^{1,4}$, Omar Ndir², Souleymane Mboup ${ }^{5}$, Sarah K Volkman ${ }^{1,3,6}$, Dyann F Wirth ${ }^{1,3^{*}}$ and Daouda Ndiaye ${ }^{2}$

\begin{abstract}
Background: Malaria treatment efforts are hindered by the rapid emergence and spread of drug resistant parasites. Simple assays to monitor parasite drug response in direct patient samples (ex vivo) can detect drug resistance before it becomes clinically apparent, and can inform changes in treatment policy to prevent the spread of resistance.

Methods: Parasite drug responses to amodiaquine, artemisinin, chloroquine and mefloquine were tested in approximately 400 Plasmodium falciparum malaria infections in Thiès, Senegal between 2008 and 2011 using a DAPI-based ex vivo drug resistance assay. Drug resistance-associated mutations were also genotyped in pfcrt and pfmdr1.

Results: Parasite drug responses changed between 2008 and 2011, as parasites became less sensitive to amodiaquine, artemisinin and chloroquine over time. The prevalence of known resistance-associated mutations also changed over time. Decreased amodiaquine sensitivity was associated with sustained, highly prevalent mutations in pfcrt, and one mutation in pfmdr1 - Y184F - was associated with decreased parasite sensitivity to artemisinin.
\end{abstract}

Conclusions: Directly measuring ex vivo parasite drug response and resistance mutation genotyping over time are useful tools for monitoring parasite drug responses in field samples. Furthermore, these data suggest that the use of amodiaquine and artemisinin derivatives in combination therapies is selecting for increased drug tolerance within this population.

Keywords: Drug resistance monitoring, DAPI ex vivo assay, Malaria

\section{Background}

Plasmodium falciparum malaria has an enormous public health impact, infecting millions and killing hundreds of thousands of people each year [1]. Drug resistance further magnifies the burden of this disease, as resistant malaria parasites have been selected by nearly every anti-malarial drug used to date. Reports of parasites with reduced susceptibility to artemisinin combination therapy (ACT) [2,3] underscore the importance of closely

\footnotetext{
* Correspondence: dfwirth@hsph.harvard.edu

${ }^{\dagger}$ Equal contributors

'Department of Immunology and Infectious Diseases, Harvard School of Public Health, Boston 02115, USA

${ }^{3}$ Infectious Disease Initiative, Broad Institute, Cambridge 02141, USA

Full list of author information is available at the end of the article
}

monitoring parasite drug responses and optimizing control strategies to quickly identify and prevent the spread of resistant parasites, particularly on the African continent [4].

Malaria drug resistance monitoring involves directly measuring parasite drug responses, or indirectly measuring the prevalence of resistance-associated mutations within a parasite population. Ex vivo drug resistance assays measure drug response in parasites taken directly from infected patients, without prior culture adaptation. These assays allow the components of combination therapies to be tested individually against parasites, and they can detect decreases in drug efficacy before resistance becomes clinically evident and widespread [5]. Many

\section{Biomed Central}


assays have been developed to test parasite drug resistance in both laboratory and field settings [6-13]. In addition, mutations in a number of parasite genetic loci have been shown to contribute to anti-malarial drug resistance, including $p f c r t$ and $p f m d r 1$, among others [14]. Monitoring the prevalence of these mutations consistently over several years can reveal trends in allele selection within a population over time, and can extend the therapeutic life of current and future treatments $[15,16]$.

The motivation for this study was to ask whether the malaria parasites circulating in Thiès, Senegal were becoming more or less resistant to anti-malarial drugs over time, and whether changes in parasite drug response could be explained by known drug resistance-associated mutations. Drug use in Senegal changed from chloroquine monotherapy to sulphadoxine, pyrimethamine and amodiaquine in 2003, and again to ACT (predominantly artesunate-amodiaquine in Thiès) in 2006 [17]. Previous drug resistance monitoring efforts in Senegal have focused on directly testing parasite drug sensitivity $[11,18]$, measuring the prevalence of resistance-associated mutations $[19,20]$, or both. The aim of this study was to measure both parasite drug sensitivity and resistance mutation prevalence over time, in order to understand how parasites in Senegal may be changing in response to drug treatment.

\section{Methods}

\section{Study population}

Individuals seeking treatment for uncomplicated $P$. falciparum malaria at the Section de Lutte Antiparasitaire (SLAP) clinic in Thiès, Senegal, during the fall transmission seasons of 2008-2011 were tested for malaria infection by microscopy and rapid diagnostic test (RDT). Plasmodium falciparum-positive patients were eligible for screening if they met the criteria described below and the patient or legal guardian provided informed written consent or assent. Eligibility criteria were: patients older than two years, axillary temperature above $37.5^{\circ} \mathrm{C}$ or history of fever within the preceding 24 hours, infection with only $P$. falciparum, no recent antimalarial drug use, and a haemoglobin level greater than $6 \mathrm{~g} / \mathrm{dL}$. Patients with symptoms of severe malaria were excluded and referred to the Thiès regional hospital for appropriate care. Study protocols and informed consent documents were approved by the Institutional Review Boards of the Senegal Ministry of Health IRB Committee and the Harvard School of Public Health (Senegal Protocol \#16330; Harvard Protocol \#P10256-127).

Among the 831 patients with uncomplicated malaria screened at the SLAP clinic between 2008 and 2011, a subset of 397 patient samples were tested for parasite drug response using the DAPI ex vivo assay (Table 1 ). The subset of patients that were tested was comparable
Table 1 Clinical parameters in screened patients and the subset tested using the DAPI ex vivo assay

\begin{tabular}{cccc}
\hline & $\begin{array}{c}\text { All screened } \\
\text { patients }\end{array}$ & $\begin{array}{c}\text { DAPI tested } \\
\text { patients }\end{array}$ & $\boldsymbol{P}$ \\
\hline Number & 831 & 397 & - \\
Gender (\% male) & 66 & 64 & 0.59 \\
Age (years) & $20(15,28)$ & $20(14,26)$ & 0.24 \\
Weight $(\mathrm{kg})$ & $55(42,65)$ & $55(39,65)$ & 0.46 \\
Temperature $\left({ }^{\circ} \mathrm{C}\right)$ & $38.2(37.2,39.7)$ & $38.4(37.3,40.0)$ & 0.25 \\
Haematocrit $(\%)$ & $38(32,40)$ & $38(32,40)$ & 0.79 \\
Parasitaemia $(\%)$ & $0.50(0.20,1.00)$ & $0.61(0.40,1.10)$ & $<.0001$ \\
\hline
\end{tabular}

Median values (with interquartile ranges) are reported for age, weight, temperature, haematocrit and parasitaemia. $P$-values were calculated using Pearson $\mathrm{X}^{2}$ for categorical variables, and Wilcox rank-sum test for continuous variables.

to the larger set of screened patients with respect to demographic parameters (age, gender) and clinical characteristics (temperature, haematocrit, weight) with the exception of parasitaemia.

\section{Sample collection and DAPI ex vivo testing}

From each subject, 5-10 mL venous blood were collected and processed on the same day. Approximately $1 \mathrm{~mL}$ of blood was spotted onto Whatman FTA ${ }^{\mathrm{mm}}$ filter paper cards for subsequent DNA extraction; the remaining blood was spun at $1,500 \mathrm{rpm}$ for 10 minutes, plasma and buffy coat were removed, and infected red blood cells were washed twice with unsupplemented RPMI media. Aliquots of each sample were cryopreserved in Glycerolyte 57 (Fenwal) supplemented with $\mathrm{AB}^{+}$serum, for subsequent culture adaptation and in vitro repeat drug testing.

Parasites were drug tested using the previously described DAPI ex vivo assay [11]. Briefly, $180 \mu \mathrm{L}$ of supplemented RPMI media containing parasitized erythrocytes at $2 \%$ haematocrit were distributed into 96 -well plates preloaded with $20 \mu \mathrm{L}$ serial dilutions of amodiaquine (USP 1031004), artemisinin (Sigma A5430), chloroquine (Sigma C6628) and mefloquine (Sigma M2319). Drug concentrations ranged from less than $1 \mathrm{nM}$ to greater than $1 \mu \mathrm{M}$ and each plate included 6-8 negative control wells with media only. Plates contained two wells of each drug concentration, were prepared in a single batch, and were frozen prior to use. When possible, samples with parasitaemia greater than $1 \%$ were diluted into leukocyte-free donor $\mathrm{O}^{+}$erythrocytes to a final plating parasitaemia of $0.4-1 \%$.

Parasites were cultured $48-72$ hours at $37^{\circ} \mathrm{C}$ under standard gas conditions $\left(1 \% \mathrm{O}_{2}, 5 \% \mathrm{CO}_{2}, 94 \% \mathrm{~N}_{2}\right)$ before addition of 4',6-diamidino-2-phenylindole (DAPI) solution, as described previously $[8,11]$. Data were collected by measuring relative fluorescence units (RFUs) using a Fluoroskan plate reader (Thermo Scientific; ex $358 \mathrm{~nm}$, 
em $461 \mathrm{~nm}$ ). 3D7 parasites were tested on each batch of drug plates to control for batch variation, and there were no consistent trends toward increased resistance among parasites tested later each season, suggesting minimal batch degradation.

\section{DNA extraction, clonality of infection and HRM genotyping}

DNA was extracted from 4-5 circular $6 \mathrm{~mm}$ punches of blood preserved on Whatman FTA $^{\mathrm{T} M}$ filter paper cards using either a QIAmp DNA Blood Mini Kit (Qiagen) or a Maxwell DNA IQ Casework Sample Kit (Promega). Parasite genomic DNA was quantified by quantitative Real-Time PCR (qPCR) [21], and clonality of infection, defined as monoclonal or polyclonal, was assessed using the 24-SNP molecular barcode [21].

High resolution melt (HRM) technology was used to genotype a set of single nucleotide polymorphisms (SNPs) associated with reduced drug sensitivity [19]. Mutations were detected based on changes in DNA sequence; in the text, mutations are referred to by the corresponding amino acid changes. Briefly, $0.01 \mathrm{ng}$ of parasite template, as quantified by qPCR [21], was used for each $5 \mu \mathrm{L}$ reaction, which included $2.5 \times$ LightScanner Master Mix with LCGreen Plus dsDNA dye (BioFire Diagnostics, Inc.). HRM analysis and genotype determination was performed on a LightScanner-384 (BioFire Diagnostics, Inc.). The HRM method can determine genotypes from as little as 10 pg of parasite DNA and can detect mutant alleles present at less than 1\% [19].

\section{Culture adaptation and in vitro drug testing}

To assess whether ex vivo drug responses were reproducible in vitro, 16 parasite isolates derived from monoclonal infections collected in 2009 were culture adapted and re-tested in vitro. Culturing was conducted under standard conditions [22] with gentle shaking at $55 \mathrm{rpm}$. Parasites were in vitro drug tested against a panel of known anti-malarials using a standard hypoxanthine incorporation assay [7], or a SYBR Green I-based drug assay [23] with modifications for 384-well format.

\section{Calculation of $\mathrm{IC}_{50}$ values and data exclusion}

Fluorescence data from drug assays were analysed using GraphPad Prism (San Diego, CA) through a fourparameter, log-logistic nonlinear regression of fluorescence intensity versus $\log _{10}$-transformed drug concentrations. To include control wells with no drug in the analysis, $1 \mathrm{nM}$ was added to each concentration value. Dose-response curves were visually inspected for fit of the sigmoidal dose-response model. Among 397 patient samples tested using the DAPI ex vivo assay, 25 samples were considered assay failures due to no parasite growth or assay contamination and were excluded from further analysis. An additional two patient samples with a plating parasitaemia below $0.1 \%$ and 30 samples with a plating parasitaemia above $1.5 \%$ were excluded, because there was no clear association between plating parasitaemia and fluorescence intensity in the no-drug wells, perhaps due to insufficient growth or saturation. This left 340 patient samples from which parasite response to at least one anti-malarial drug was determined.

Drug curves that did not exhibit the standard sigmoidal dose-response shape were classified as either fitting an exponential or linear decay model, and had their $\mathrm{IC}_{50}$ values estimated through these alternative models, or were excluded. When $\mathrm{IC}_{50}$ values from technical replicates could not be estimated due to a single outlier point, this point was excluded.

\section{Data and statistical analysis}

Dynamic range of the DAPI ex vivo assay was assessed by calculating the signal-to-noise ratio (SNR) and Z'-factor of each assay. SNR was measured by dividing fluorescence signal (RFUs) from no-drug wells by fluorescence signal from maximum drug wells. The median signal-to-noise ratio (SNR) among all assays was 3:1 (Interquartile Range $=2: 1,5: 1)$. Z'-factor was calculated using the following equation: $Z^{\prime}=1-[(3$ standard deviations of positive controls +3 standard deviations of negative controls)/ absolute difference between negative and positive controls] [24]. The median Z'-factor among all assays was 0.61 ; Z'-factors greater than 0 are considered acceptable, and $Z$ '-factors greater than 0.5 are considered excellent [24]. Reliability of the DAPI ex vivo assay was measured by evaluating agreement between technical replicates in the untransformed scale using the intraclass correlation coefficient (ICC) for agreement. Only sigmoidal curves were analysed, to avoid biases due to lack of fitness of different $\mathrm{IC}_{50}$ curve-fitting models. For all drugs, mean differences in $\mathrm{IC}_{50}$ values between replicates were approximately zero, as expected. Except for a few outliers (fewer than five points for each drug), differences between replicates were small compared with the $\mathrm{IC}_{50}$ range of each drug.

Statistical analyses were performed in GraphPad Prism (v5.0d, San Diego, CA) and R-2.11.1. IC C $_{50}$ values measured ex vivo were compared to in vitro $\mathrm{IC}_{50}$ values from culture-adapted parasites by calculating the intraclass correlation coefficient (ICC) for consistency ( $\mathrm{R}$ package irr), and by linear regression. To monitor population drug sensitivity, $\mathrm{IC}_{50}$ variations over time were measured through linear regression with $\log _{10}$-transformed $\mathrm{IC}_{50}$ values. Primary analysis focused on non-linear trends (using indicator variables for years) but results were confirmed by assessing linear trends. Multiple regression models were used to measure whether $\mathrm{IC}_{50}$ values changed significantly over time after accounting for the effect of potential 
confounders (clonality, haematocrit, parasitaemia, age, and temperature). Since parasites with reduced drug sensitivity may first arise in subpopulations that exhibit larger $\mathrm{IC}_{50}$ values, the $90^{\text {th }}$ percentile among all $\mathrm{IC}_{50}$ values is reported for each year. Changes in the prevalence of drug resistance markers over time were measured by FisherHamilton exact test, and 95\% confidence intervals for marker prevalence are based on the logit ( $R$ package

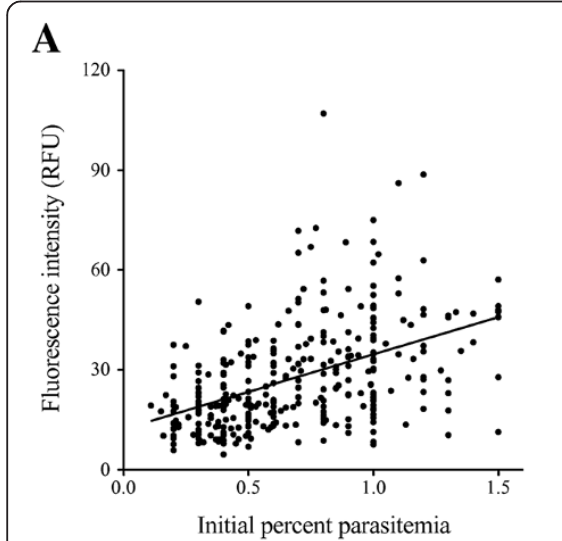

D

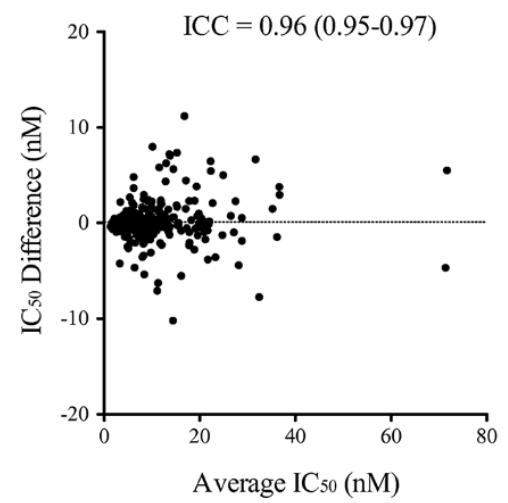

G

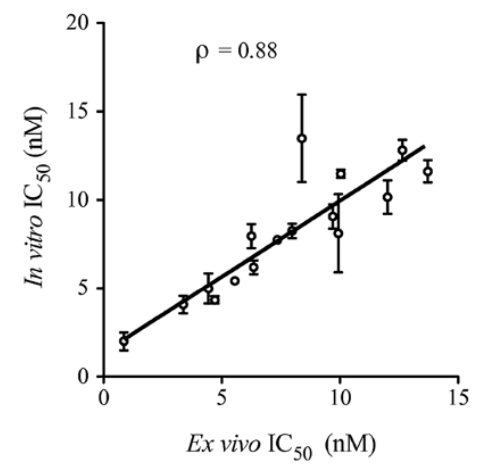

B

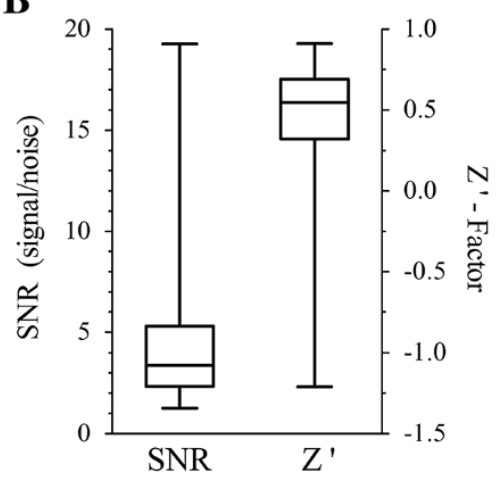

E

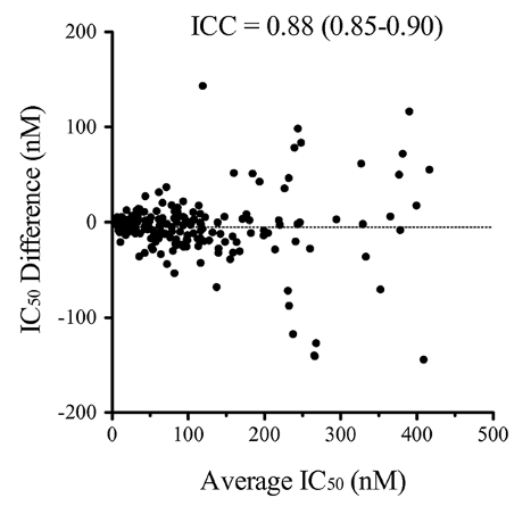

H

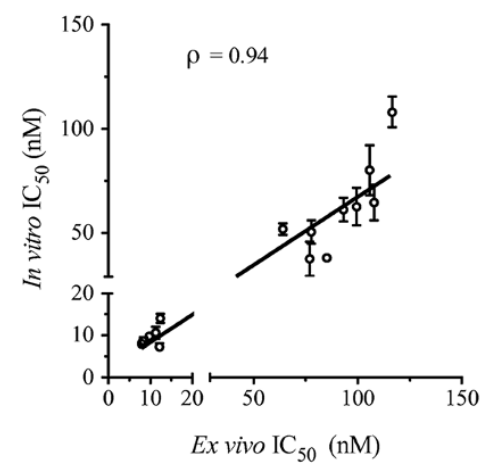

C

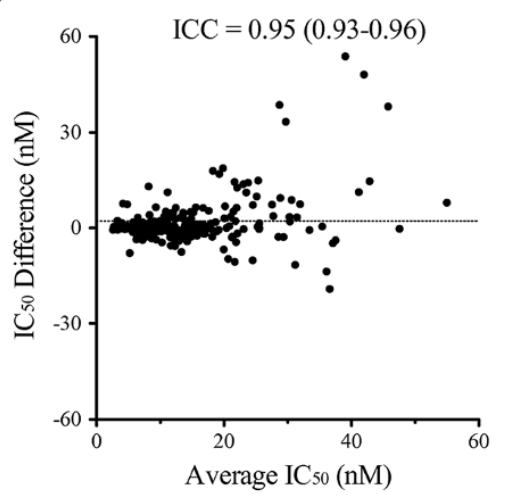

F

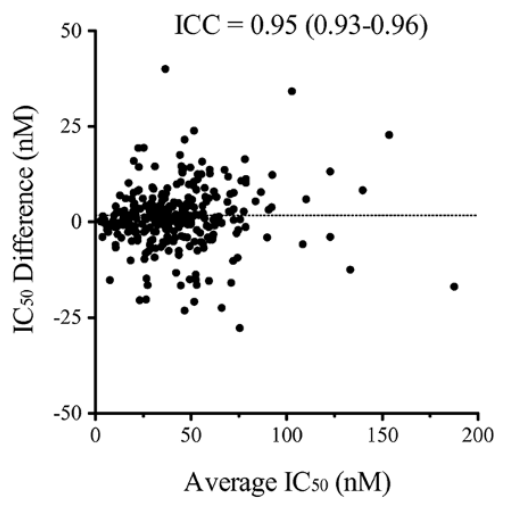

I

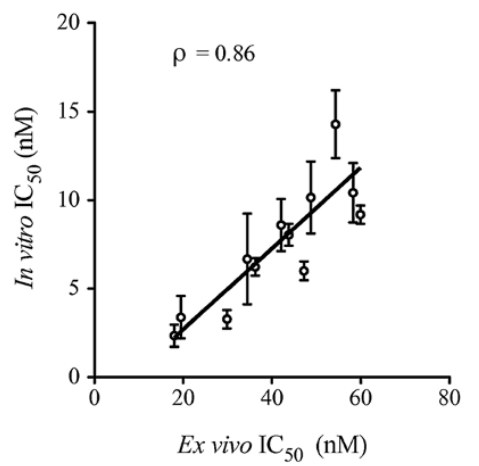

Figure 1 Validation of the DAPI ex vivo drug assay. A. Fluorescence intensity of maximum growth wells versus initial parasitaemia for parasites tested in the DAPI ex vivo drug assay. Pearson $\rho=0.47$, linear slope $P<0.0001$. B. Box plots showing signal-to-noise ratio (SNR) and Z'-factor for all assays. C-F. Bland-Altman plots showing differences between $I C_{50}$ values of each technical replicate vs. average $I C_{50}$ values for amodiaquine (C), artemisinin (D), chloroquine (E), and mefloquine (F). Horizontal lines indicate the mean difference in $I_{50}$ values between replicates. Intra-class correlation coefficients (ICC, with corresponding 95\% confidence intervals) are displayed on each graph. G-I. Comparison of ex vivo with in vitro $I_{50}$ values for artemisinin $(\mathbf{G})$, chloroquine $\mathbf{( H )}$, and mefloquine $\mathbf{( I )}$, among culture-adapted monoclonal parasites collected in 2009. Mean in vitro $I_{50}$ values are plotted with error bars showing the standard error of at least two biological replicates. $\rho$ denotes the Pearson correlation coefficient. 
binom). Finally, associations between $\mathrm{IC}_{50}$ values and the occurrence of drug resistance-associated mutations in pfcrt and pfmdr1 were assessed through the Wilcoxon rank-sum test.

\section{Results}

\section{DAPI ex vivo assay validation}

The usefulness of the DAPI-based ex vivo assay for monitoring drug sensitivity among the parasites circulating in Thiès, Senegal was assessed by measuring the dynamic range and reliability of the assay. This ex vivo assay could be used to accurately calculate anti-malarial 50 -percent inhibitory concentrations $\left(\mathrm{IC}_{50}\right.$ values $)$ in direct patient samples, as evidenced by the dynamic range of the assay (Figure 1A and B), and by testing for reproducibility between technical replicates (Figure 1C-F). A subset of parasites derived from monoclonal infections was culture adapted and retested for their susceptibility to chloroquine, mefloquine and artemisinin in vitro (Figure 1G-I). In vitro drug responses were highly correlated with ex vivo responses, with systematic differences in scale presumably due to the technical differences between assays. Amodiaquine was not included in the in vitro testing because all parasites tested were sensitive to the drug, and the range of observed $\mathrm{IC}_{50}$ values was narrow. Overall, the DAPI ex vivo assay provided valid and consistent results, and could, therefore, be used as a tool to directly measure malaria parasite drug responses in patient samples.

\section{Distribution of parasite drug responses across years}

The DAPI ex vivo drug assay was used to compare parasite drug responses to amodiaquine, artemisinin, chloroquine and mefloquine among all parasites tested between
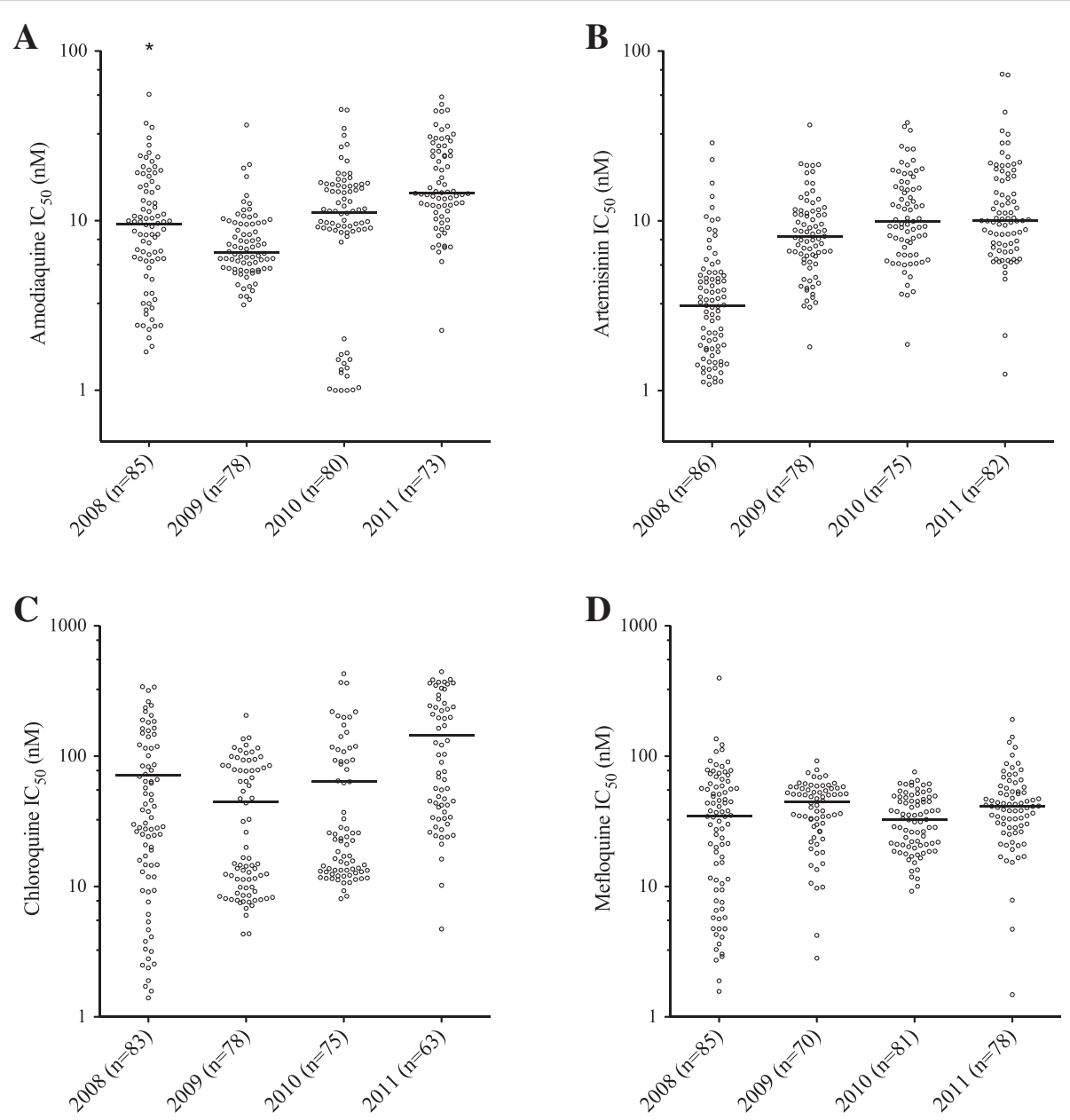

Figure 2 Changes in ex vivo parasite sensitivity over time. $I C_{50}$ values among parasites collected in Thiès, Senegal and tested against amodiaquine (A), artemisinin (B), chloroquine (C), and mefloquine (D). The number of samples tested each year is indicted in parentheses below each plot. Horizontal lines indicate median $I C_{50}$ values. The asterisk in panel $\mathbf{A}$ indicates an $I C_{50}$ value off the scale (amodiaquine $I C_{50}=1140 \mathrm{nM}$ ). 
2008 and 2011 (Figure 2, Table 2). During this time, parasite $\mathrm{IC}_{50}$ values increased for amodiaquine, artemisinin and chloroquine $(P<0.001$ for linear and non-linear trends and in both crude and adjusted analyses). Parasite $\mathrm{IC}_{50}$ values for mefloquine also changed, but after adjusting for confounders only a non-linear trend in drug responses over time was detected. The $90^{\text {th }}$ percentile $\mathrm{IC}_{50}$ values, representing the most resistant parasites observed each year, also increased between 2008 and 2011 for amodiaquine, artemisinin and chloroquine (Table 2). Finally, there was a large range of parasite responses to amodiaquine and artemisinin, two anti-malarials that have been used in combination therapies in this area since 2006 [17]. $\mathrm{IC}_{50}$ values ranged from $1 \mathrm{nM}$ to over $50 \mathrm{nM}$ for amodiaquine (except for one outlier in 2008), and from $1 \mathrm{nM}$ to over $70 \mathrm{nM}$ for artemisinin.

\section{Prevalence of resistance-associated mutations over time}

In addition to directly testing parasite drug responses, known drug resistance-associated mutations in pfcrt and $p f m d r 1$ were genotyped in order to assess changes in the prevalence of these mutations over time. No change in the prevalence of the mutant haplotype at protein positions 72-76 within pfcrt was detected (Figure 3A). This haplotype was almost always inherited along with the A220S mutation in pfcrt, and both mutations remained

Table 2 Parasite drug responses measured in the DAPI ex vivo assay

\begin{tabular}{|c|c|c|c|c|}
\hline & $\begin{array}{c}2008 \\
(N=86)\end{array}$ & $\begin{array}{c}2009 \\
(N=78)\end{array}$ & $\begin{array}{c}2010 \\
(N=81)\end{array}$ & $\begin{array}{c}2011 \\
(N=82)\end{array}$ \\
\hline \multicolumn{5}{|l|}{ Amodiaquine } \\
\hline Median $I_{50}$ & 9.6 & 6.5 & 11.2 & 14.5 \\
\hline$I_{50}$ Range & $1.6,1140$ & $3.2,36.7$ & $1.0,45.3$ & $2.3,53.7$ \\
\hline $90^{\text {th }}$ percentile $\mid C_{50}$ & 24.0 & 11.8 & 22.2 & 35.4 \\
\hline \multicolumn{5}{|l|}{ Artemisinin } \\
\hline Median $\mathrm{IC}_{50}$ & 3.2 & 8.1 & 9.9 & 10.1 \\
\hline $\mathrm{IC}_{50}$ Range & $1.1,28.8$ & $1.8,36.7$ & $1.9,38.0$ & $1.3,73.1$ \\
\hline $90^{\text {th }}$ percentile $I C_{50}$ & 9.3 & 17.0 & 22.4 & 24.9 \\
\hline \multicolumn{5}{|l|}{ Chloroquine } \\
\hline Median $\mathrm{IC}_{50}$ & 30.7 & 15.0 & 22.4 & 76.1 \\
\hline$I_{50}$ Range & $1.4,341.5$ & $4.3,205.5$ & $8.1,430.2$ & $4.7,455.0$ \\
\hline $90^{\text {th }}$ percentile $I C_{50}$ & 199.4 & 108.2 & 199.1 & 364.6 \\
\hline \multicolumn{5}{|l|}{ Mefloquine } \\
\hline Median $\mathrm{IC}_{50}$ & 34.5 & 44.6 & 32.6 & 41.2 \\
\hline$I_{50}$ Range & $1.6,398.0$ & $2.8,92.1$ & $9.2,75.7$ & $1.5,191.5$ \\
\hline $90^{\text {th }}$ percentile $I_{50}$ & 84.6 & 62.5 & 59.0 & 82.4 \\
\hline
\end{tabular}

$\mathrm{IC}_{50}$ values are in $\mathrm{nM}$. IC $\mathrm{C}_{50}$ Range $=$ minimum $\mathrm{IC} \mathrm{C}_{50}$, maximum $\mathrm{IC}_{50} \cdot 90^{\text {th }}$ percentile $I C_{50}=I C_{50}$ value of the $90^{\text {th }}$ percentile within the range of drug responses observed each year. above $50 \%$ prevalence in this population, in contrast to other studies within Senegal [20], and elsewhere in Africa [25]. Prevalence of the N326S mutation, however, did change over time $(P<0.05)$, and was not linked to the other typed mutations within $p f c r t$.

While the mutations typed in pfcrt either stayed the same or increased in prevalence over time, the mutations typed in pfmdr1 showed two distinct patterns in their prevalence over the four years studied (Figure 3B). The N86Y and N1042D mutations both decreased in prevalence between 2008 and $2011(P<0.05)$. By 2011, the N86Y mutation was detected in fewer than $5 \%$ of parasites, and the N1042D mutation was absent from the population sampled in both 2010 and 2011. Conversely, the Y184F mutation more than doubled in prevalence, from approximately $30 \%$ in 2008 to greater than $70 \%$ in $2011(P<0.05)$.

\section{Correlations between resistance mutations and ex vivo drug responses}

To assess whether the use of amodiaquine and artemisinin derivatives could be driving the observed changes in resistance mutation prevalence, associations between mutations and $\mathrm{IC}_{50}$ values were examined. The occurrence of mutant genotypes at pfcrt 72-76, A220S, and N326S were all associated with increased amodiaquine $\mathrm{IC}_{50}$ values (Table 3 ). These same pfcrt mutations were also associated with higher chloroquine $\mathrm{IC}_{50}$ values, possibly due to cross-resistance between chloroquine and amodiaquine (Pearson $\rho=0.6$ ). The mutant $72-76$ haplotype and A220S were also associated with artemisinin sensitivity, and none of the typed pfcrt mutations were associated with mefloquine response.

Associations between mutations in pfmdr1 and parasite drug response were also detected. Parasites with wild-type alleles at amino acid positions 86 and 1042, as well as parasites with the mutant allele at position 184, had increased artemisinin $\mathrm{IC}_{50}$ values (Table 3 ). Artemisinin was the only drug associated with the Y184F mutation in this population - this mutation was not associated with amodiaquine, chloroquine, or mefloquine responses. No significant association was seen between amodiaquine or chloroquine responses and any of the mutations typed in $p f m d r 1$, in contrast to previous findings [26-28], and perhaps due to a small number of parasites possessing the N86Y and N1042D mutations. Stratifying first on the pfcrt 72-76 haplotype did not reveal an association between amodiaquine or chloroquine and any of the pfmdr1 mutations that were genotyped. Finally, higher mefloquine $\mathrm{IC}_{50}$ values were also associated with the wild-type allele at position 86 within pfmdr1; this was the only significant association between any of the typed mutations and mefloquine $\mathrm{IC}_{50}$ values. 


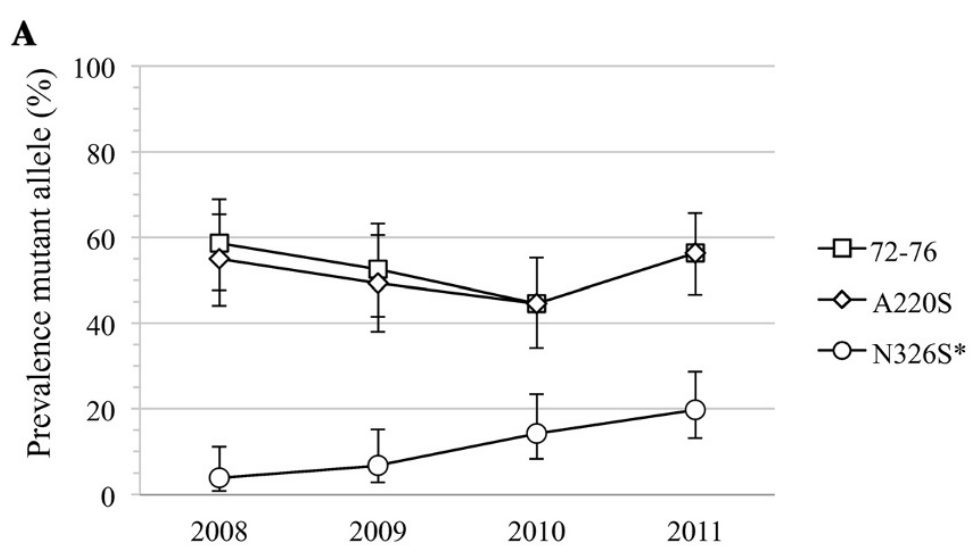

B

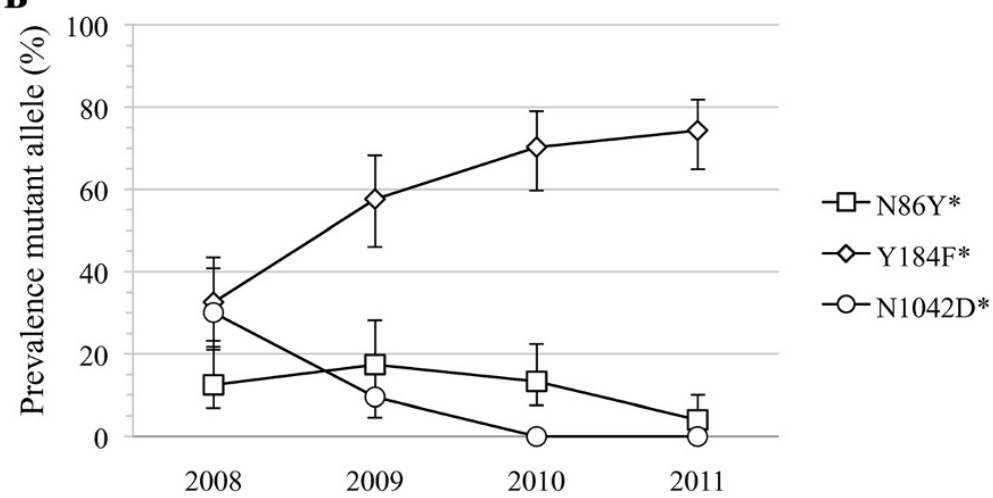

Figure 3 Changes in prevalence of known drug resistance-associated mutations over time. Prevalence of resistance-associated mutations in pfcrt (A) and pfmdri (B) with corresponding 95\% point-wise confidence intervals. Mutations were measured by high-resolution melt (HRM) technology and prevalence was calculated by dividing the number of samples containing at least one mutant allele by the total number of samples genotyped each year. Asterisks indicate significant changes over time $(P<0.05$ by Fisher-Hamilton exact test).

\section{Discussion}

Ex vivo assays are an important tool for malaria drug resistance monitoring in direct patient samples. These assays complement in vivo studies by allowing researchers to test parasite responses to different drugs individually and in the absence of patient factors that might introduce noise or confound results. Importantly, ex vivo monitoring of malaria parasite drug responses can provide an early warning of decreased parasite sensitivity before parasites become highly resistant and cause infected patients to fail drug treatment.

The DAPI ex vivo assay performed well, with excellent agreement between technical replicates, good dynamic range, and very good correlation between drug responses measured ex vivo with those measured in vitro. Furthermore, the $\mathrm{IC}_{50}$ values observed in Thiès, Senegal between 2008 and 2011 were comparable to other ex vivo studies of $P$. falciparum drug response [10,18,29]. Because parasite drug responses were measured over a four-year time span, trends in drug response in this population over time could also be assessed. The trends observed in parasite responses to amodiaquine and artemisinin suggest that malaria parasites in Thiès are becoming more tolerant to these compounds. Data from future years of ex vivo monitoring will be critical in determining whether these trends continue.

The trends observed in the prevalence of resistanceassociated mutations in pfcrt and pfmdrl suggest that anti-malarial drug use is selecting for resistanceassociated alleles within this population. In contrast to other studies $[11,20,25]$, resistance-associated mutations within $p f c r t$ remained prevalent within this population, and even appeared to increase in prevalence between 2010 and 2011. This suggests either that compensatory mutations have restored the fitness of resistant parasites, and/or that anti-malarial drug use is maintaining these mutations within the population. The continuous distribution of chloroquine $\mathrm{IC}_{50}$ values observed in 2008 and 2011 further suggests that additional mutations affecting parasite drug response exist in this population. The finding that parasites with mutations in pfcrt have higher amodiaquine $\mathrm{IC}_{50}$ values is in agreement with previous studies of laboratory parasite lines [26], and malariainfected patients [28]. Additionally, amodiaquine has been 
Table 3 Associations between wild-type and mutant genotypes and ex vivo drug responses

\begin{tabular}{|c|c|c|c|c|c|c|c|c|c|c|c|c|}
\hline \multirow[b]{3}{*}{$\begin{array}{l}\text { Allele } \\
\qquad N_{(w t)} / N_{(m u t)}\end{array}$} & \multicolumn{12}{|c|}{$\begin{array}{l}\text { Median } \mathrm{IC}_{50} \text { Values } \\
\text { (Interquartile Range) }\end{array}$} \\
\hline & \multicolumn{3}{|c|}{ Amodiaquine } & \multicolumn{3}{|c|}{ Artemisinin } & \multicolumn{3}{|c|}{ Chloroquine } & \multicolumn{3}{|c|}{ Mefloquine } \\
\hline & Wild-type & Mutant & $P$ & Wild-type & Mutant & $P$ & Wild-type & Mutant & $P$ & Wild-type & Mutant & $P$ \\
\hline pfcrt $72-76^{a}$ & 9 & 12 & 0.004 & 9 & 7 & 0.005 & 14 & 99 & $<0.0001$ & 42 & 36 & 0.3 \\
\hline $160 / 182$ & $(6,15)$ & $(8,19)$ & & $(6,15)$ & $(4,10)$ & & $(11,28)$ & $(53,196)$ & & $(21,57)$ & $(21,52)$ & \\
\hline pfcrt A220S & 9 & 12 & 0.0005 & 9 & 7 & 0.01 & 14 & 99 & $<0.0001$ & 42 & 36 & 0.3 \\
\hline $163 / 174$ & $(6,14)$ & $(8,19)$ & & $(6,15)$ & $(4,10)$ & & $(11,28)$ & $(53,196)$ & & $(21,57)$ & $(21,52)$ & \\
\hline pfcrt N326S & 10 & 15 & 0.01 & 8 & 9 & 0.6 & 28 & 139 & $<0.0001$ & 38 & 38 & 0.7 \\
\hline $295 / 40$ & $(7,16)$ & $(9,20)$ & & $(5,13)$ & $(5,12)$ & & $(13,93)$ & $(84,217)$ & & $(21,54)$ & $(28,56)$ & \\
\hline pfmdr1 N86Y & 10 & 9 & 0.6 & 8 & 5 & 0.0002 & 33 & 75 & 0.4 & 41 & 17 & $<0.0001$ \\
\hline $296 / 37$ & $(7,16)$ & $(6,17)$ & & $(5,13)$ & $(3,8)$ & & $(14,101)$ & $(12,201)$ & & $(26,57)$ & $(9,22)$ & \\
\hline pfmdr1 Y184F & 10 & 11 & 0.1 & 7 & 8 & 0.01 & 29 & 34 & 0.6 & 41 & 36 & 0.3 \\
\hline $136 / 202$ & $(6,16)$ & $(7,16)$ & & $(4,11)$ & $(6,14)$ & & $(12,109)$ & $(13,114)$ & & $(21,58)$ & $(21,52)$ & \\
\hline pfmdr1 N1042D & 10 & 8 & 0.1 & 8 & 4 & $<0.0001$ & 33 & 47 & 1.0 & 38 & 38 & 0.9 \\
\hline $307 / 31$ & $(7,16)$ & $(5,16)$ & & $(6,13)$ & $(2,5)$ & & $(13,109)$ & $(15,115)$ & & $(21,53)$ & $(18,59)$ & \\
\hline
\end{tabular}

$\mathrm{IC}_{50}$ values are in $\mathrm{nM} . \mathrm{N}_{(\mathrm{wt})}=$ number of samples possessing only the wild-type allele. $\mathrm{N}_{(\mathrm{mut})}=$ number of samples possessing at least one mutant allele. Mixed genotypes were detected at the following prevalence: pfcrt 72-76 $(n=22) ; p f c r t$ A220S $(n=19) ; p f c r t$ N326S $(n=4) ; p f m d r 1$ N86Y $(n=7) ; p f m d r 1$ Y184F $(n=22) ; p f m d r 1$ N1042D $(n=1)$. Genotypes were determined by high-resolution melt (HRM) genotyping. $P$-values were calculated using the Wilcoxon rank-sum test, and associations where $P<0.05$ are shown in bold.

a pfcrt protein positions $72-76$ were all perfectly correlated and were analysed as a haplotype rather than individually.

administered to malaria-infected patients in Senegal since 2003 [17]. Because increased amodiaquine $\mathrm{IC}_{50}$ values were associated with the typed $p f c r t$ mutations, it seems possible that use of amodiaquine is preserving these mutations within the population.

The observed trends in resistance mutations within $p f m d r 1$ suggest that artemisinin compounds are selecting for a combination of wild-type and mutant alleles within this gene. The N86 and 184F alleles have been previously associated with in vivo selection by ACT [30,31], and two recent studies of the prevalence of drug resistance markers in Dakar, Senegal also found a high prevalence of the Y184F mutation [32,33]. Furthermore, Y184F has been found to be under selection among parasite populations in Cambodia [34], where artemisinin resistance, defined as delayed parasite clearance, has been described. While the artemisinin resistance phenotype of delayed in vivo parasite clearance does not appear to correlate well with ex vivo or standard in vitro assays [2], artemisinin resistance might occur through different mechanisms in Africa as compared to southeast Asia. Furthermore, as parasites become increasingly artemisinin resistant in vivo, they may become amenable to monitoring with ex vivo assays such as this one. The disappearance of the N86Y and N1042D mutations, coupled with the rapid rise of the Y184F mutation, suggest that selective pressure is acting on $p f m d r 1$, eliminating some mutations while driving others to high prevalence within this population. Because artemisinin response was associated with all three of the typed pfmdr1 mutations, it appears that the artemisinin derivatives used in ACT might be the selective force driving the Y184F mutation to high prevalence, while simultaneously selecting for the wild-type alleles at positions 86 and 1042.

These findings are consistent with the hypothesis that amodiaquine use in Thiès, Senegal has selected for chloroquine resistance-associated mutations within pfcrt, while artemisinin compounds have selected for a particular combination of wild-type and mutant alleles within pfmdr1. In both cases, alleles that make parasites better able to withstand drug pressure are likely selected. Other African countries that have used artesunate-amodiaquine have also documented sustained high prevalence of chloroquine resistance-associated mutations within pfcrt [35,36]. Conversely, countries deploying $\mathrm{ACT}$ that does not include amodiaquine have seen a return to chloroquine-sensitivity after chloroquine was removed from the treatment arsenal [25,37], presumably due to the fitness costs of resistanceassociated mutations. Other African countries have also documented recent increases in the prevalence of the pfmdr1 N86 and 184F alleles [38,39], though this study marks the highest recorded prevalence to date of the Y184F mutation on the African continent.

\section{Conclusions}

Ex vivo monitoring of malaria parasite drug response is a powerful tool for malaria control. Directly testing 
parasite drug responses and genotyping resistanceassociated mutations can provide early warnings of decreased parasite sensitivity to the individual drugs used in combination therapies, before parasites become highly resistant and cause infected patients to fail drug treatment. Ex vivo drug assays such as the one used here could also provide phenotype data for analyses aimed at identifying additional drug resistance markers [40-42]. As ACT is the first-line treatment throughout Africa, the ability to detect resistance to both artemisinin derivatives and partner compounds as they emerge will become increasingly important in order to preserve the efficacy of these drugs.

\section{Competing interests}

The authors declare that they have no competing interests.

\section{Authors' contributions}

DVT, PDS, ON, SM, SKV, DFW and DN designed the experiments. DVT, BD, RFD, PDS, AKL, MN, AKB, YDN, EJH and SKV carried out the experiments and collected data. DVT, RFD, CV, PDS and SKV analysed the data. DVT, CV, SKV, DFW and DN wrote the manuscript. All authors read and approved the final manuscript.

\section{Authors' information}

Daria Van Tyne and Baba Dieye are equal contributors (co-first authors). Clarissa Valim and Rachel F Daniels are equal contributors (co-second authors).

\section{Acknowledgements}

We gratefully acknowledge members of the sample collection team at the SLAP clinic in Thiès, including Younous Diedhiou, Lamine Ndiaye, Amadou Mactar Mbaye, Ngayo Sy and Moussa Dieng Sarr. We also thank Meaghan Galligan, Justin Becker, Kate Fernandez, and Vishal Patel for technical assistance and thoughtful discussions. This work was supported by the National Institutes of Health [R01 Al075080-01A1 and D43 TW001503], and by the ExxonMobil Foundation.

\section{Author details}

'Department of Immunology and Infectious Diseases, Harvard School of Public Health, Boston 02115, USA. 'Laboratory of Parasitology and Mycology, Université Cheikh Anta Diop, BP5005, Dakar, Senegal. Infectious Disease Initiative, Broad Institute, Cambridge 02141, USA. ${ }^{4}$ Department of Human and Evolutionary Biology, Harvard University, Cambridge 02138, USA. ${ }^{5}$ Laboratory of Bacteriology and Virology, Université Cheikh Anta Diop, Dakar BP5005, Senegal. ${ }^{6}$ School of Nursing and Health Sciences, Simmons College, Boston 02115, USA.

Received: 6 July 2013 Accepted: 24 November 2013 Published: 6 December 2013

\section{References}

1. Alonso PL, Brown G, Arevalo-Herrera M, Binka F, Chitnis C, Collins F, Doumbo OK, Greenwood B, Hall BF, Levine MM, Mendis K, Newman RD, Plowe CV, Rodriguez MH, Sinden R, Slutsker L, Tanner M: A research agenda to underpin malaria eradication. PLoS Med 2011, 8:e1000406.

2. Noedl H, Se Y, Schaecher K, Smith BL, Socheat D, Fukuda MM, Consortium ARiCAS: Evidence of artemisinin-resistant malaria in western Cambodia. NEJM 2008, 359:2619-2620.

3. Dondorp AM, Nosten F, Yi P, Das D, Phyo AP, Tarning J, Lwin KM, Ariey F, Hanpithakpong W, Lee SJ, Ringwald P, Silamut K, Imwong M, Chotivanich K, Lim P, Herdman T, An SS, Yeung S, Singhasivanon P, Day NPJ, Lindegardh $\mathrm{N}$, Socheat D, White NJ: Artemisinin resistance in Plasmodium falciparum malaria. NEJM 2009, 361:455-467.

4. Talisuna AO, Karema C, Ogutu B, Juma E, Logedi J, Nyandigisi A, Mulenga M, Mbacham WF, Roper C, Guerin PJ, D'Alessandro U, Snow RW: Mitigating the threat of artemisinin resistance in Africa: improvement of drug- resistance surveillance and response systems. Lancet Infect Dis 2012, 12:888-896.

5. Noedl H, Wongsrichanalai C, Wernsdorfer WH: Malaria drug-sensitivity testing: new assays, new perspectives. Trends Parasitol 2003, 19:175-181.

6. Rieckmann KH, Campbell GH, Sax LJ, Ema JE: Drug sensitivity of Plasmodium falciparum: an in vitro microtechnique. Lancet 1978, 311:22-23.

7. Desjardins RE, Canfield CJ, Haynes JD, Chulay JD: Quantitative assessment of antimalarial activity in vitro by a semiautomated microdilution technique. Antimicrob Agents Chemother 1979, 16:710-718.

8. Baniecki ML, Wirth DF, Clardy J: High-throughput Plasmodium falciparum growth assay for malaria drug discovery. Antimicrob Agents Chemother 2007, 51:716-723.

9. Bennett TN, Paguio M, Gligorijevic B, Seudieu C, Kosar AD, Davidson E, Roepe PD: Novel, rapid, and inexpensive cell-based quantification of antimalarial drug efficacy. Antimicrob Agents Chemother 2004, 48:1807-1810.

10. Rason MA, Randriantsoa T, Andrianantenaina H, Ratsimbasoa A, Menard D: Performance and reliability of the SYBR Green I based assay for the routine monitoring of susceptibility of Plasmodium falciparum clinical isolates. Trans R Soc Trop Med Hyg 2008, 102:346-351.

11. Ndiaye D, Patel V, Demas A, LeRoux M, Ndir O, Mboup S, Clardy J, Lakshmanan V, Daily JP, Wirth DF: A non-radioactive DAPI-based high-throughput in vitro assay to assess Plasmodium falciparum responsiveness to antimalarials-increased sensitivity of $P$. falciparum to chloroquine in Senegal. Am J Trop Med Hyg 2010, 82:228-230.

12. Druilhe P, Moreno A, Blanc C, Brasseur PH, Jacquier P: A colorimetric in vitro drug sensitivity assay for Plasmodium falciparum based on a highly sensitive double-site lactate dehydrogenase antigen-capture enzyme-linked immunosorbent assay. Am J Trop Med Hyg 2001, 64:233-241.

13. Noedl H, Attlmayr B, Wernsdorfer WH, Kollaritsch H, Miller RS: A histidinerich protein 2-based malaria drug sensitivity assay for field use. Am J Trop Med Hyg 2004, 71:711-714.

14. Wilson PE, Alker AP, Meshnick SR: Real-time PCR methods for monitoring antimalarial drug resistance. Trends Parasitol 2005, 21:278-283.

15. Sibley $\mathrm{CH}$, Ringwald P: A database of antimalarial drug resistance. Malar $J$ 2006, 5:48.

16. Vestergaard LS, Ringwald P: Responding to the challenge of antimalarial drug resistance by routine monitoring to update national malaria treatment policies. Am J Trop Med Hyg 2007, 77:153-159.

17. WHO: Focus on Senegal. In Roll Back Malaria Progress and Impact Series, Volume 4. Geneva: WHO; 2010:1-56.

18. Fall B, Diawara S, Sow K, Baret E, Diatta B, Fall KB, Mbaye PS, Fall F, Dieme Y, Rogier C, Wade B, Bercion R, Pradines B: Ex vivo susceptibility of Plasmodium falciparum isolates from Dakar, Senegal, to seven standard anti-malarial drugs. Malar J 2011, 10:310.

19. Daniels R, Ndiaye D, Wall M, McKinney J, Sene PD, Sabeti PC, Volkman SK, Mboup S, Wirth DF: Rapid, field-deployable method for genotyping and discovery of drug-resistance single nucleotide polymorphisms in Plasmodium falciparum. Antimicrob Agents Chemother 2012, 56(6):2976-2986.

20. Ndiaye M, Faye B, Tine R, Ndiaye JL, Lo A, Abiola A, Dieng Y, Ndiaye D, Hallett R, Alifrangis M, Gaye O: Assessment of the molecular marker of Plasmodium falciparum chloroquine resistance ( $p f c r t)$ in Senegal after several years of chloroquine withdrawal. Am J Trop Med Hyg 2012, 87(4):640-645

21. Daniels R, Volkman SK, Milner DA, Mahesh N, Neafsey DE, Park DJ, Rosen D, Angelino E, Sabeti PC, Wirth DF, Wiegand RC: A general SNP-based molecular barcode for Plasmodium falciparum identification and tracking. Malar J 2008, 7:223.

22. Trager $W$, Jensen JB: Human malaria parasites in continuous culture. Science 1976, 193:673-675.

23. Plouffe D, Brinker A, McNamara C, Henson K, Kato N, Kuhen K, Nagle A, Adrian F, Matzen JT, Anderson P, Nam TG, Gray NS, Chatterjee A, Janes J, Yan SF, Trager R, Caldwell JS, Schultz PG, Zhou Y, Winzeler EA: In silico activity profiling reveals the mechanism of action of antimalarials discovered in a high-throughput screen. Proc Natl Acad Sci U S A 2008, 105:9059-9064.

24. Zhang JH, Chung TD, Oldenburg KR: A simple statistical parameter for use in evaluation and validation of high throughput screening assays. J Biomol Screen 1999, 4:67-73. 
25. Mita T, Kaneko A, Lum JK, Bwijo B, Takechi M, Zungu IL, Tsukahara T, Tanabe K, Kobayakawa T, Bjorkman A: Recovery of chloroquine sensitivity and low prevalence of the Plasmodium falciparum chloroquine resistance transporter gene mutation K76T following the discontinuance of chloroquine use in Malawi. Am J Trop Med Hyg 2003, 68:413-415.

26. Sa JM, Twu O, Hayton K, Reyes S, Fay MP, Ringwald P, Wellems TE: Geographic patterns of Plasmodium falciparum drug resistance distinguished by differential responses to amodiaquine and chloroquine. Proc Natl Acad Sci U S A 2009, 106:18883-18889.

27. Picot S, Olliaro P, de Monbrison F, Bienvenu AL, Price RN, Ringwald P: A systematic review and meta-analysis of evidence for correlation between molecular markers of parasite resistance and treatment outcome in falciparum malaria. Malar J 2009, 8:89.

28. Happi CT, Gbotosho GO, Folarin OA, Bolaji OM, Sowunmi A, Kyle DE, Milhous W, Wirth DF, Oduola AM: Association between mutations in Plasmodium falciparum chloroquine resistance transporter and $P$. falciparum multidrug resistance 1 genes and in vivo amodiaquine resistance in P. falciparum malaria-infected children in Nigeria. Am J Trop Med Hyg 2006, 75:155-161.

29. Pascual A, Parola P, Benoit-Vical F, Simon F, Malvy D, Picot S, Delaunay P, Basset D, Maubon D, Faugere B, Menard G, Bourgeois N, Oeuvray C, Didillon $E$, Rogier C, Pradines B: Ex vivo activity of the ACT new components pyronaridine and piperaquine in comparison with conventional ACT drugs against isolates of Plasmodium falciparum. Malar J 2012, 11:45.

30. Dokomajilar C, Nsobya SL, Greenhouse B, Rosenthal PJ, Dorsey G: Selection of Plasmodium falciparum pfmdr1 alleles following therapy with artemether-lumefantrine in an area of Uganda where malaria is highly endemic. Antimicrob Agents Chemother 2006, 50:1893-1895.

31. Gadalla NB, Adam I, Elzaki SE, Bashir S, Mukhtar I, Oguike M, Gadalla A, Mansour F, Warhurst D, El-Sayed BB, Sutherland CJ: Increased pfmdr1 copy number and sequence polymorphisms in Plasmodium falciparum isolates from Sudanese malaria patients treated with artemether-lumefantrine. Antimicrob Agents Chemother 2011, 55:5408-5411.

32. Wurtz N, Fall B, Pascual A, Diawara S, Sow K, Baret E, Diatta B, Fall KB, Mbaye PS, Fall F, Dieme Y, Rogier C, Bercion R, Briolant S, Wade B, Pradines B: Prevalence of molecular markers of Plasmodium falciparum drug resistance in Dakar, Senegal. Malar J 2012, 11:197.

33. Fall B, Pascual A, Sarr FD, Wurtz N, Richard V, Baret E, Dieme Y, Briolant S, Bercion R, Wade B, Tall A, Pradines B: Plasmodium falciparum susceptibility to anti-malarial drugs in Dakar, Senegal, in 2010: an ex vivo and drug resistance molecular markers study. Malar J 2013, 12:107.

34. Vinayak S, Alam MT, Sem R, Shah NK, Susanti Al, Lim P, Muth S, Maguire JD, Rogers WO, Fandeur T, Barnwell JW, Escalante AA, Wongsrichanalai C, Ariey F, Meshnick SR, Udhayakumar V: Multiple genetic backgrounds of the amplified Plasmodium falciparum multidrug resistance (pfmdr1) gene and selective sweep of 184F mutation in Cambodia. J Infect Dis 2010, 201:1551-1560.

35. Frank M, Lehners N, Mayengue PI, Gabor J, Dal-Bianco M, Kombila DU, Ngoma GM, Supan C, Lell B, Ntoumi F, Grobusch MP, Dietz K, Kremsner PG: A thirteen-year analysis of Plasmodium falciparum populations reveals high conservation of the mutant pfcrt haplotype despite the withdrawal of chloroquine from national treatment guidelines in Gabon. Malar J 2011, 10:304.

36. Menard S, Morlais I, Tahar R, Sayang C, Mayengue PI, Iriart X, Benoit-Vical F, Lemen B, Magnaval JF, Awono-Ambene P, Basco LK, Berry A: Molecular monitoring of Plasmodium falciparum drug susceptibility at the time of the introduction of artemisinin-based combination therapy in Yaounde, Cameroon: implications for the future. Malar J 2012, 11:113.

37. Mwai L, Ochong E, Abdirahman A, Kiara SM, Ward S, Kokwaro G, Sasi P, Marsh K, Borrmann S, Mackinnon M, Nzila A: Chloroquine resistance before and after its withdrawal in Kenya. Malar J 2009, 8:106.

38. Thomsen $T$, Ishengoma DS, Mmbando BP, Lusingu JP, Vestergaard LS, Theander TG, Lemnge MM, Bygbjerg IC, Alifrangis M: Prevalence of single nucleotide polymorphisms in the Plasmodium falciparum multidrug resistance gene (pfmdr1) in Korogwe District in Tanzania before and after introduction of artemisinin-based combination therapy. Am J Trop Med Hyg 2011, 85:979-983.

39. Lekana-Douki JB, Dinzouna Boutamba SD, Zatra R, Zang Edou SE, Ekomy H, Bisvigou U, Toure-Ndouo FS: Increased prevalence of the Plasmodium falciparum pfmdr1 86N genotype among field isolates from Franceville,
Gabon after replacement of chloroquine by artemether-lumefantrine and artesunate-mefloquine. Infect Genet Evol 2011, 11:512-517.

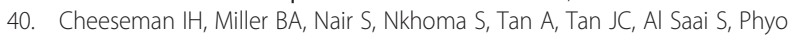
AP, Moo CL, Lwin KM, McGready R, Ashley E, Imwong M, Stepniewska K, Y P, Dondorp AM, Mayxay M, Newton PN, White NJ, Nosten F, Ferdig MT, Anderson TJ: A major genome region underlying artemisinin resistance in malaria. Science 2012, 336:79-82.

41. Park DJ, Lukens AK, Neafsey DE, Schaffner SF, Chang HH, Valim C, Ribacke U, Van Tyne D, Galinsky K, Galligan M, Becker JS, Ndiaye D, Mboup S, Wiegand RC, Hartl DL, Sabeti PC, Wirth DF, Volkman SK: Sequence-based association and selection scans identify drug resistance loci in the Plasmodium falciparum malaria parasite. Proc Natl Acad Sci U S A 2012, 109(32):13052-13057.

42. Takala-Harrison S, Clark TG, Jacob CG, Cummings MP, Miotto O, Dondorp AM, Fukuda MM, Nosten F, Noedl H, Imwong M, Bethell D, Se Y, Lon C, Tyner SD, Saunders DL, Socheat D, Ariey F, Phyo AP, Starzengruber $P$, Fuehrer HP, Swoboda P, Stepniewska K, Flegg J, Arze C, Cerqueira GC, Silva JC, Ricklefs SM, Porcella SF, Stephens RM, Adams M, et al: Genetic loci associated with delayed clearance of Plasmodium falciparum following artemisinin treatment in Southeast Asia. Proc Natl Acad Sci U S A 2013, 110:240-245.

\section{doi:10.1186/1475-2875-12-441}

Cite this article as: Van Tyne et al.: Changes in drug sensitivity and antimalarial drug resistance mutations over time among Plasmodium falciparum parasites in Senegal. Malaria Journal 2013 12:441.

\section{Submit your next manuscript to BioMed Central and take full advantage of:}

- Convenient online submission

- Thorough peer review

- No space constraints or color figure charges

- Immediate publication on acceptance

- Inclusion in PubMed, CAS, Scopus and Google Scholar

- Research which is freely available for redistribution 